\title{
How Many Passes Are Needed for Endobronchial Ultrasound-Guided Transbronchial Needle Aspiration for Sarcoidosis? A Prospective Multicenter Study
}

\author{
Masahide Oki ${ }^{a}$ Hideo Saka $^{a}$ Masahiko Ando ${ }^{b}$ Harunori Nakashima ${ }^{c}$ \\ Akira Shiraki $^{c}$ Yasushi Murakami ${ }^{a}$ Yoshihito Kogure $^{\mathrm{a}}$ Chiyoe Kitagawa $^{\mathrm{a}}$ \\ Tatsuo Kato $^{d}$ \\ a Department of Respiratory Medicine, Nagoya Medical Center, Nagoya, Japan; ${ }^{b}$ Center for Advanced Medicine \\ and Clinical Research, Nagoya University Hospital, Nagoya, Japan; ' Department of Respiratory Medicine, Ogaki

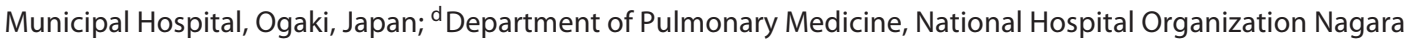 \\ Medical Center, Gifu, Japan
}

\section{Keywords}

Bronchoscopy · Endobronchial ultrasound · Rapid on-site cytological evaluation · Sarcoidosis · Transbronchial needle aspiration

\begin{abstract}
Background: While endobronchial ultrasound-guided transbronchial needle aspiration (EBUS-TBNA) is widely used as an initial diagnostic procedure for pathological confirmation of sarcoidosis, it is unclear how many passes are required to obtain diagnostic materials. Objectives: The aim of this study was to determine the number of needle passes needed for the diagnosis of stage $\mathrm{I} / \mathrm{II}$ sarcoidosis using EBUSTBNA. Methods: At three institutions, 109 patients with suspected stage I/II sarcoidosis were recruited and underwent 6 passes of EBUS-TBNA for the main target lesion. Additional EBUS-TBNA for other lesions was permitted. The cumulative yields of needle passes for detecting noncaseating epithelioid cell granulomas were analyzed. Results: A total of 109 patients underwent EBUS-TBNA for 184 lesions. EBUS-TBNA
\end{abstract}

identified specimens containing granulomas in 81 of 92 patients (88\%) with a final diagnosis of sarcoidosis. The cumulative yields through the first, second, third, fourth, fifth, and sixth passes for the main target lesion were $63,75,82,85,86$ and $88 \%$, respectively. In the 55 patients that underwent EBUS-TBNA for multiple lesions, the cumulative yields of 2 passes per lesion for 2 lesions (total of 4 passes) and of 4 passes for single lesions were 86 and $84 \%$, respectively ( $p=$ $1.00)$. Conclusions: If rapid on-site cytological evaluation is not available, we recommend at least 4 passes per patient for either single or multiple lesions with EBUS-TBNA for pathological diagnosis of stage $\mathrm{I} / \mathrm{Il}$ sarcoidosis.

(C) 2018 S. Karger AG, Basel

\section{Introduction}

Sarcoidosis is a systematic multiorgan disorder of unknown etiology [1]. The diagnosis has been made by pathological confirmation of noncaseating epithelioid cell granulomas, as well as clinicoradiological compatibil-
(C) 2018 S. Karger AG, Basel
Masahide Oki

Department of Respiratory Medicine, Nagoya Medical Center

4-1-1 Sannomaru, Naka-ku

Nagoya 460-0001 (Japan)

E-Mail masahideo@aol.com 
ity. As lung and intrathoracic lymph nodes are invaded in most cases, bronchoscopy has been widely used for sampling specimens to confirm granulomas [1]. Traditionally, transbronchial lung biopsy, endobronchial biopsy, and transbronchial needle aspiration have been considered as standard procedures. However, over the last decade, a number of innovative bronchoscopic instruments have been developed and techniques have been improved. Endobronchial ultrasound-guided transbronchial needle aspiration (EBUS-TBNA) is one of the most innovative bronchoscopic procedures, which has significantly changed the evaluation of hilar-mediastinal lesions [2]. Since the first report in 2007 [3], there have been many subsequent reports regarding the usefulness of EBUSTBNA for diagnosing sarcoidosis [4-12]. Therefore, if available, the use of EBUS-TBNA has been recommended for sampling specimens in patients with suspected sarcoidosis $[13,14]$.

Although there have been many reports regarding the usefulness of EBUS-TBNA for the diagnosis of sarcoidosis, a uniform methodology, with respect to the number of needle passes to obtain a sufficient diagnostic yield, has not been established $[13,14]$. The present study was performed to determine the number of needle passes needed for the diagnosis of stage I/II sarcoidosis using EBUSTBNA.

\section{Patients and Methods}

\section{Patients}

This prospective study was carried out at three institutions in Japan (Nagoya Medical Center, Nagara Medical Center, and Ogaki Municipal Hospital). From January 2013 to March 2015, a total of 109 patients with suspected stage I (lymphadenopathy on chest roentgenogram) or stage II (lymphadenopathy and lung parenchymal changes on chest roentgenogram) sarcoidosis were enrolled. The study population consisted of patients with suspected stage I/II sarcoidosis based on clinicoradiological pictures with hilar-mediastinal nodes $\geq 10 \mathrm{~mm}$ in short-axis diameter on computed tomography (CT), and in whom the attending physician considered bronchoscopic biopsy necessary for management decision. Patients with pathologically proven sarcoidosis were excluded. This prospective study was approved by the institutional review board of each institution and registered with the University Hospital Medical Information Network-Clinical Trials Registry (identifier: UMIN000009753). Informed consent was obtained from all patients prior to enrollment.

\section{Procedures}

EBUS-TBNA was performed using an ultrasound bronchoscope (BF-UC260FW; Olympus; Tokyo, Japan) in combination with an ultrasound processor (EU-ME1; Olympus) under local anesthesia and conscious sedation. The ultrasound bronchoscope was passed through the mouth to the trachea, and then an ultrasound transducer covered with a saline-filled balloon was brought into contact with the airway wall and moved in all directions to identify the lesions for sampling. Once the target lesion was visualized by ultrasound imaging, needle aspiration using a 22-G needle (NA-201SX-4022; Olympus) was performed under real-time ultrasound guidance. The aspirated specimens within the needle were pushed out by a needle stylet and then blown by air from a syringe onto a glass slide. The visible tissue fragments on the glass slide were then collected and transferred into separate containers filled with formalin for histological examination, and the remaining specimen on the glass slide was immediately smeared and fixed in $95 \%$ alcohol for cytological examination. The residual specimen stored at the lumen of the needle and catheter was then washed and flushed into saline for culture for microbiological analysis including acid-fast bacilli and fungi. Rapid on-site cytological evaluation (ROSE) was not performed according to the purposes of the study. EBUS-TBNA was performed with 6 passes for the main target lymph node, for which easily accessible, suspicious, and large lymph nodes were selected. Further bronchoscopic procedures, including additional punctures for other lymph nodes, transbronchial biopsy, and bronchoalveolar lavage, were permitted if the operator considered it necessary.

\section{Diagnosis}

Each histological and cytological specimen was interpreted separately regarding compatibility of sarcoidosis by the local pathologist at each institution. Sarcoidosis was cytologically diagnosed using the criteria of Tambouret et al. [15], as follows: wellformed granulomas characterized by tightly aggregated groups of at least 10 histiocytes with ovoid-to-elongate, often twisted nuclei, and moderately abundant amphophilic cytoplasm. The histiocyte shape varies from epithelioid to spindle-shaped. The granuloma size varies from small to large, but tends to be uniform. The number of granulomas also varies from few to many. Multinucleated giant cells and single epithelioid histiocytes are often present in association with the granulomas.

The final diagnosis of sarcoidosis was made by the attending physician based on the clinicoradiological compatibility at the time of the procedures and during the clinical follow-up period, as well as on the pathological findings of noncaseating granulomas, and with the exclusion of other causes of granuloma.

\section{Endpoints}

The primary outcome of this study was the number of needle passes needed for the diagnosis of stage I/II sarcoidosis using EBUS-TBNA. The secondary outcome was the diagnostic yield and safety of EBUS-TBNA.

\section{Statistical Analysis}

In patients with a positive EBUS-TBNA result for sarcoidosis, ascertaining the number of passes needed to achieve a cumulative yield of $95 \%$ was assumed appropriate. We estimated that at least 76 EBUS-TBNA-proven sarcoidosis patients would be required under the following conditions based on a binomial distribution: alternative yield of $95 \%$, null yield of $85 \%$, and statistical power of $90 \%$ at a one-sided significance level of 0.05 . We expected EBUSTBNA to provide specimens with granulomas in about $80 \%$ of patients, and thus the target number of participants was set to 90 . If the number of patients with granulomas did not reach 76 at the 
Table 1. Characteristics of patients and lesions

\begin{tabular}{lc}
\hline Characteristics & Data \\
\hline Number of patients & 109 \\
Median age (range), years & $58(24-82)$ \\
Sex & \\
$\quad$ Male & $61(56)$ \\
Female & $48(44)$ \\
Chest radiographic staging & \\
Stage I & $58(53)$ \\
Stage II & $51(47)$ \\
Serum angiotensin-converting enzyme & \\
$\quad \leq 25.0$ U/L & $88(81)$ \\
$>25.0$ U/L & $21(19)$ \\
Total LN number evaluated by EBUS-TBNA & 184 \\
LN number evaluated by EBUS-TBNA per patient & \\
1 LN & $36(33)$ \\
2 LNs & $71(65)$ \\
3 LNs & $2(2)$ \\
LN location for EBUS-TBNA & \\
2R & $1(1)$ \\
$4 \mathrm{R}$ & $59(32)$ \\
$4 \mathrm{~L}$ & $5(3)$ \\
7 & $92(50)$ \\
$10 \mathrm{R}$ & $3(2)$ \\
$10 \mathrm{~L}$ & $2(1)$ \\
$11 \mathrm{R}$ & $16(9)$ \\
$11 \mathrm{~L}$ & $6(3)$ \\
Median LN size in shortest diameter (range), & \\
mm & $15.7(7.6-33.0)$ \\
\hline & \\
& \\
& \\
&
\end{tabular}

Data are presented as $n$ (\%) unless indicated otherwise. EBUSTBNA, endobronchial ultrasound-guided transbronchial needle aspiration; LN, lymph node.
Table 2. Diagnostic performance of EBUS-TBNA

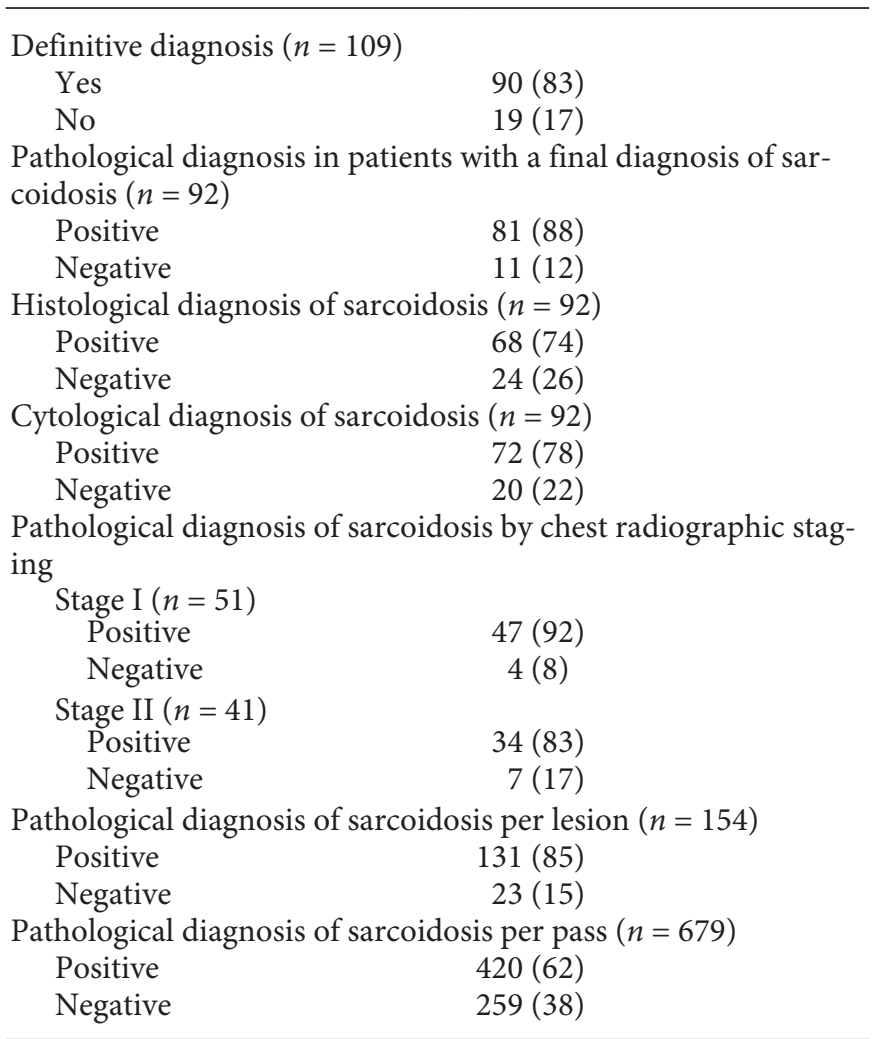

Figures are given as $n(\%)$. EBUS-TBNA, endobronchial ultrasound-guided transbronchial needle aspiration; LN, lymph node. time of enrollment of 90 patients, we aimed to continue recruiting patients until $\geq 76$ patients with granulomas had been enrolled.

Statistical analyses were performed using PASW Statistics (ver. 18.0; SPSS Inc., Chicago, IL, USA). Means and percentages are presented as appropriate. Categorical variables were analyzed using the Fisher exact test or the Pearson $\chi^{2}$ test. For the comparison of cumulative yields, the odds ratio (OR) and $p$ value were calculated by generalized estimating equations. The Tukey-Kramer test was used to adjust for multiple testing. In all analyses, $p<0.05$ was taken to indicate statistical significance.

\section{Results}

In the 109 patients enrolled in this study, we performed 816 passes for 184 lymph nodes, with a median shortest diameter of $15.7 \mathrm{~mm}$ on chest CT. The characteristics of the patients and lesions are shown in Table 1. Six passes for the main target could be performed for 106 of 109 patients (97\%); only 5 passes could be performed in 2 patients due to cough and involuntary movement caused by conscious sedation, and only 4 passes could be performed in 1 patient due to water leakage from the balloon.

Figure 1 presents a patient selection flow chart, and Table 2 shows the diagnostic performance of EBUSTBNA. EBUS-TBNA provided diagnostic materials in 90 of 109 patients $(83 \%$; $95 \%$ confidence interval [CI]: $74-$ $89 \%$ ). EBUS-TBNA did not result in significant complications, with only minor issues, including coughing and involuntary movement, as described above, occurring during the procedures. A final diagnosis of sarcoidosis was made in 92 patients with a median follow-up of 841 days (range: 48-1,329 days), and 84 patients had sarcoidosis with pathological confirmation. In patients with a final diagnosis of sarcoidosis, EBUS-TBNA provided cytological and/or histological specimens with epithelioid cell 
Fig. 1. Patient selection flow chart. EBUSTBNA, endobronchial ultrasound-guided transbronchial needle aspiration.

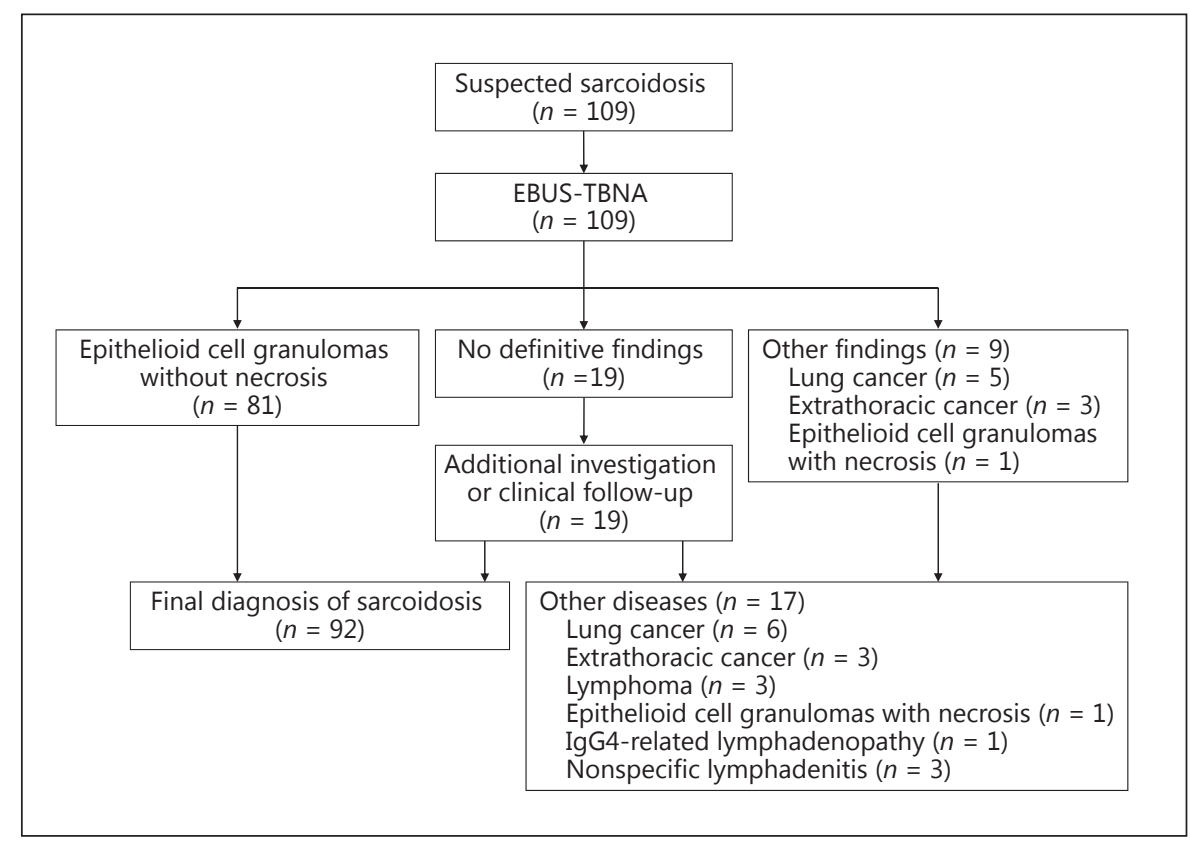

granulomas in 81 of 92 patients (88\%), 131 of 154 lesions (85\%), and 420 specimens obtained with 679 passes (62\%). Thus, the sensitivity of EBUS-TBNA for pathological diagnosis of sarcoidosis was 88\% (95\% CI: 80 94\%, stage I; 47 of 51 patients [92\%, 95\% CI: 81-98\%], stage II; 34 of 41 patients [83\%, 95\% CI: 68-93\%], $p=$ 0.21 ). The sensitivity of the diagnoses in the three institutions did not differ (institution 1; 89\% [51 of 57 patients], institution 2; 85\% [17 of 20 patients], institution 3; 87\% [13 of 15 patients], $p=0.855$ ).

The cumulative yields of EBUS-TBNA, for the main target lesion by the number of needle passes in 92 patients with a final diagnosis of sarcoidosis, are shown in Table 3. The cumulative yields through the first, second, third, fourth, fifth, and sixth passes for the main target lesion were $63,75,82,85,86$, and $88 \%$, respectively. The cumulative yield of EBUS-TBNA increased significantly after 2 passes versus 1 pass ( $\mathrm{OR}=1.73,95 \% \mathrm{CI}: 1.27-2.36, p=$ $0.007)$. The difference in cumulative yield between 2 and 4 passes was also significant $(\mathrm{OR}=1.81,95 \% \mathrm{CI}$ : 1.45 $2.63, p=0.02$ ). The cumulative yield moderately increased after 5 or 6 passes, but the difference was not significant ( 4 vs. 5 passes, $\mathrm{OR}=1.09,95 \%$ CI: $0.93-1.27, p=0.92 ; 4$ vs. 6 passes, $\mathrm{OR}=1.30,95 \% \mathrm{CI}$ : $0.97-1.74, p=0.49$ ).

In the 55 patients that underwent at least 2 passes of EBUS-TBNA for the second target lesion, the cumulative yields of 2 passes per lesion for 2 lesions (total of 4 passes) and of 4 passes for single lesions were $86 \%$ (47 of 55 patients) and $84 \%$ ( 46 of 55 patients), respectively $(p=1.00)$.

\section{Discussion}

This multicenter prospective study demonstrated that at least 4 passes per patient for either single or multiple lymph nodes with EBUS-TBNA are necessary to obtain a sufficient diagnostic yield for patients with suspected stage I/II sarcoidosis.

In EBUS-TBNA, a deficiency in the number of passes results in insufficient diagnostic yield, while an excessive number of passes leads to a greater amount of time being spent on sampling by the operator, and interpretation of specimens by the pathologist. Furthermore, patients may be at risk of complications, even though EBUS-TBNA is a safe procedure. Lee et al. [16] investigated the number of needle passes needed for mediastinal staging of non-small lung cancer in EBUS-TBNA. EBUS-TBNA with 4 passes per lymph node was performed in 163 mediastinal lymph node stations in 102 patients with potentially operable non-small cell lung cancer. The sensitivities for differentiating between malignant and benign lymph node stations were $69.8,83.7,95.3$, and $95.3 \%$ for $1,2,3$, and 4 passes, respectively. Based on the results of Lee et al. [16], a recent guideline [14] on the technical aspects of EBUS-TBNA suggests that a minimum of 3 separate needle passes should be performed per sampling site in patients with suspected lung cancer and who are undergoing EBUS-TBNA for diagnosis (if ROSE is not used). Unfortunately, there are no data regarding the number of needle passes required for diseases other than lung cancer in EBUS-TBNA [14]. 
Table 3. Cumulative yield of EBUS-TBNA according to the number of needle passes in 92 patients with sarcoidosis

\begin{tabular}{|c|c|c|c|}
\hline Number of passes & Patients af & dle pass, $n(\%)$ & \\
\hline 1 & $58(63.0)$ & \multirow{3}{*}{$\begin{array}{l}\mathrm{OR}=1.73(p=0.0065) \\
\mathrm{OR}=1.45(p=0.1188)\end{array}$} & \multirow{6}{*}{$\mathrm{OR}=1.81(p=0.0230)$} \\
\hline 2 & $69(75.0)$ & & \\
\hline 3 & $75(81.5)$ & & \\
\hline 4 & $78(84.8)$ & \multirow{3}{*}{$\mathrm{OR}=1.09(p=0.9164)$} & \\
\hline 5 & $79(85.9)$ & & \\
\hline 6 & $81(88.0)$ & & \\
\hline
\end{tabular}

EBUS-TBNA is recommended for the diagnosis of sarcoidosis $[13,14]$, but no optimal methodology (in terms of specimen handling, or the use or non-use of ROSE) has yet been established. Some investigators $[3,8]$ employed techniques similar to ours, whereas others $[7,11]$ used liquid-based cytological techniques. Previously $[4,9,17]$, we reported high sampling yields when 21- or 22-G needles were used to obtain core specimens for histology; combination histology/cytology afforded a better diagnostic yield than either technique alone. However, some clinicians find it difficult to obtain core histological specimens via EBUS-TBNA using fine needles [18]. Liquid-based cytological techniques applied to cell block analyses may be useful alternatives in such situations. ROSE is a reliable method for evaluating sample adequacy during EBUSTBNA. It provides valuable information for the determination of procedure termination, to reduce the puncture number or eliminate the need for additional bronchoscopic procedures $[19,20]$. It has been used mainly for differentiating between malignant and benign specimens. However, it can also be used for evaluating cytological specimens with sarcoidosis during EBUS-TBNA [18]. In a prospective study of 60 patients with suspected sarcoidosis [18], EBUS-TBNA provided cytological specimens with granulomas in 45 of 49 patients with a final diagnosis of sarcoidosis. ROSE during EBUS-TBNA provided 43 true-positive results and 1 false-positive result for diagnosing sarcoidosis. According to the high accuracy of EBUS-TBNA with ROSE, and high concordance rate between the final result and ROSE result, the authors con- cluded that EBUS-TBNA with ROSE should be considered as the first-line investigation of sarcoidosis. In fact, some studies on EBUS-TBNA for sarcoidosis used ROSE during the procedure $[3,5]$. The limitation of ROSE is its limited availability; in many institutions, ROSE cannot be used because of manpower and cost issues [21].

Some investigators have assessed the number of passes or lymph nodes required as a secondary endpoint $[5,6$, 21-23]. In an early study on the usefulness of EBUSTBNA for diagnosing sarcoidosis, Garwood et al. [5] reported a diagnostic yield of $85 \%$, and the cumulative yield exceeded $80 \%$ at 5 passes. Unfortunately, EBUS-TBNA was performed with 4 or fewer passes in the majority of cases because of the use of ROSE in their study. In addition, precise data on the number of needle passes performed per patient and lymph node were not available in their report. In a study to determine factors for diagnosing sarcoidosis by EBUS-TBNA, Sun et al. [21] investigated the diagnostic yield per lymph node according to the number of passes. In their study, 111 patients had a final diagnosis of sarcoidosis, in whom EBUS-TBNA without ROSE was performed for 284 lymph nodes. Of the 284 lymph nodes, the diagnostic yield in lymph nodes with $1,2,3,4,5,6$, and 7 passes was $45 \%$ ( 9 of 20 ), $79 \%$ (87 of 110 ), $86 \%$ ( 79 of 92 ), $85 \%$ (35 of 41 ), $93 \%$ (13 of 14 ), $67 \%$ ( 2 of 3 ), and $50 \%$ ( 2 of 4 ), respectively. The diagnostic yield in specimens with 3 passes per lymph node nearly reached a plateau, while the diagnostic yield decreased with more than 5 passes per lymph node. The authors recommended EBUS-TBNA with 3-5 passes for the larg- 
est lymph node [21]. Different from our current study, the cumulative yield per lymph node was not analyzed in the study by Sun et al. [21], as the number of needle passes per lymph node was not precisely defined. A few investigators have suggested that the use of EBUS-TBNA for multiple lymph nodes rather than a single lymph node increases the diagnostic yield $[6,22]$, but this remains controversial $[21,23]$. Our study indicated no difference in diagnostic yield between 1 and 2 lymph nodes.

We performed all EBUS-TBNA procedures under local anesthesia (i.e., conscious sedation); it is thus unclear whether our findings are relevant to procedures performed under general anesthesia. However, an earlier randomized trial [24] found no significant difference in terms of the diagnostic yield of EBUS-TBNA when similar numbers of passes were performed on patients under moderate sedation and general anesthesia. Thus, current EBUS-TBNA guidelines suggest that the nature of sedation does not influence either the specimen quality or the diagnostic yield of EBUS-TBNA [14, 25]. Although we performed EBUS-TBNA under local anesthesia (conscious sedation), our results may also be applicable when general anesthesia is applied.

The present study had some limitations. The final diagnosis of sarcoidosis was not pathologically proven in all patients, similar to the majority of other studies on sar- coidosis. In addition, not all patients (95\% of patients) with a final diagnosis of sarcoidosis could be followed up for 6 months, which was used as the follow-up period in some other studies $[6,8,11,12]$. Although each case was reviewed carefully for the possibility of other diseases, this may result in incorrect diagnosis of sarcoidosis. Another potential limitation of the present study concerns the number of passes for the main target lesions defined in our protocol. We performed EBUS-TBNA with 6 passes for the main target lesion in each patient, but the yield did not reach a plateau at up to 6 passes. Although we could not determine the number of passes that would provide the maximum yield, empirically we expect that the possibility of obtaining the first diagnostic specimen after the seventh pass would be extremely low, and thus that our results are reliable.

In conclusion, if ROSE is not used, we recommend at least 4 passes of EBUS-TBNA per patient for either single or multiple lesions for the pathological diagnosis of stage I/II sarcoidosis.

\section{Financial Disclosure and Conflicts of Interest}

The authors have no conflicts of interest to disclose.

\section{References}

1 Statement on sarcoidosis. Joint statement of the American Thoracic Society (ATS), the European Respiratory Society (ERS) and the World Association of Sarcoidosis and Other Granulomatous Disorders (WASOG) adopted by the ATS Board of Directors and by the ERS Executive Committee, February 1999. Am J Respir Crit Care Med 1999;160: 736-755.

-2 Varela-Lema L, Fernández-Villar A, RuanoRavina A: Effectiveness and safety of endobronchial ultrasound-transbronchial needle aspiration: a systematic review. Eur Respir J 2009;33:1156-1164.

3 Wong M, Yasufuku K, Nakajima T, Herth FJ, Sekine Y, Shibuya K, Iizasa T, Hiroshima K, Lam WK, Fujisawa T: Endobronchial ultrasound: new insight for the diagnosis of sarcoidosis. Eur Respir J 2007;29:1182-1186.

4 Oki M, Saka H, Kitagawa C, Tanaka S, Shimokata T, Kawata Y, Mori K, Kajikawa S, Ichihara S, Moritani S: Real-time endobronchial ultrasound-guided transbronchial needle aspiration is useful for diagnosing sarcoidosis. Respirology 2007;12:863-868.
5 Garwood S, Judson MA, Silvestri G, Hoda R, Fraig M, Doelken P: Endobronchial ultrasound for the diagnosis of pulmonary sarcoidosis. Chest 2007;132:1298-1304.

-6 Tremblay A, Stather DR, Maceachern P, Khalil M, Field SK: A randomized controlled trial of standard vs. endobronchial ultrasonography-guided transbronchial needle aspiration in patients with suspected sarcoidosis. Chest 2009; 136:340-346.

$\checkmark 7$ Tournoy KG, Bolly A, Aerts JG, Pierard P, De Pauw R, Leduc D, Leloup A, Pieters T, Slabbynck H, Janssens A, Carron K, Schrevens L, Pat K, De Keukeleire T, Dooms C: The value of endoscopic ultrasound after bronchoscopy to diagnose thoracic sarcoidosis. Eur Respir J 2010;35:1329-1335.

8 Navani N, Booth HL, Kocjan G, Falzon M, Capitanio A, Brown JM, Porter JC, Janes SM: Combination of endobronchial ultrasound guided transbronchial needle aspiration with standard bronchoscopic techniques for the diagnosis of stage I and stage II pulmonary sarcoidosis. Respirology 2011;16:467-472.
9 Oki M, Saka H, Kitagawa C, Kogure Y, Murata $\mathrm{N}$, Ichihara S, Moritani S: Prospective study of endobronchial ultrasound-guided transbronchial needle aspiration of lymph nodes versus transbronchial lung biopsy of lung tissue for diagnosis of sarcoidosis. J Thorac Cardiovasc Surg 2012;143:1324-1329.

10 Plit M, Pearson R, Havryk A, Da Costa J, Chang C, Glanville AR: Diagnostic utility of endobronchial ultrasound-guided transbronchial needle aspiration compared with transbronchial and endobronchial biopsy for suspected sarcoidosis. Intern Med J 2012;42: 434-438.

11 von Bartheld MB, Dekkers OM, Szlubowski A, Eberhardt R, Herth FJ, in't Veen JC, de Jong YP, van der Heijden EH, Tournoy KG, Claussen M, van den Blink B, Shah PL, Zoumot Z, Clementsen P, Porsbjerg C, Mauad T, Bernardi FD, van Zwet EW, Rabe KF, Annema JT: Endosonography vs. conventional bronchoscopy for the diagnosis of sarcoidosis: the GRANULOMA randomized clinical trial. JAMA 2013;309:2457-2464. 
12 Gupta D, Dadhwal DS, Agarwal R, Gupta N, Bal A, Aggarwal AN: Endobronchial ultrasound-guided transbronchial needle aspiration vs. conventional transbronchial needle aspiration in the diagnosis of sarcoidosis. Chest 2014; 146:547-556.

-13 Agarwal R, Srinivasan A, Aggarwal AN, Gupta D: Efficacy and safety of convex probe EBUS-TBNA in sarcoidosis: a systematic review and meta-analysis. Respir Med 2012; 106:883-892.

14 Wahidi MM, Herth F, Yasufuku K, Shepherd RW, Yarmus L, Chawla M, Lamb C, Casey KR, Patel S, Silvestri GA, Feller-Kopman DJ: Technical aspects of endobronchial ultrasound-guided transbronchial needle aspiration: CHEST Guideline and Expert Panel Report. Chest 2016;149:816-835.

15 Tambouret R, Geisinger KR, Powers CN, Khurana KK, Silverman JF, Bardales R, Pitman MB: The clinical application and cost analysis of fine-needle aspiration biopsy in the diagnosis of mass lesions in sarcoidosis. Chest 2000;117:1004-1011.

16 Lee HS, Lee GK, Lee HS, Kim MS, Lee JM, Kim HY, Nam BH, Zo JI, Hwangbo B: Realtime endobronchial ultrasound-guided transbronchial needle aspiration in mediastinal staging of non-small cell lung cancer: how many aspirations per target lymph node station? Chest 2008;134:368-374.
7 Oki M, Saka H, Kitagawa C, Kogure Y, Murata $\mathrm{N}$, Adachi $\mathrm{T}$, Ichihara $\mathrm{S}$, Moritani S: Transesophageal bronchoscopic ultrasoundguided fine needle aspiration for diagnosis of sarcoidosis. Respiration 2013;85:137-143.

18 Plit ML, Havryk AP, Hodgson A, James D, Field A, Carbone S, Glanville AR, Bashirzadeh F, Chay AM, Hundloe J, Pearson R, Fielding D: Rapid cytological analysis of endobronchial ultrasound-guided aspirates in sarcoidosis. Eur Respir J 2013;42:1302-1308.

19 Oki M, Saka H, Kitagawa C, Kogure Y, Murata N, Adachi T, Ando M: Rapid on-site cytologic evaluation during endobronchial ultrasound-guided transbronchial needle aspiration for diagnosing lung cancer: a randomized study. Respiration 2013;85:486-492.

20 Murakami Y, Oki M, Saka H, Kitagawa C, Kogure Y, Ryuge M, Tsuboi R, Oka S, Nakahata M, Funahashi Y, Hori K, Ise Y, Ichihara S, Moritani S: Endobronchial ultrasoundguided transbronchial needle aspiration in the diagnosis of small cell lung cancer. Respir Investig 2014;52:173-178.

21 Sun J, Yang H, Teng J, Zhang J, Zhao H, Garfield DH, Han B: Determining factors in diagnosing pulmonary sarcoidosis by endobronchial ultrasound-guided transbronchial needle aspiration. Ann Thorac Surg 2015;99: 441-445.
22 Cetinkaya E, Gunluoglu G, Ozgul A, Gunluoglu MZ, Ozgul G, Seyhan EC, Gencoglu A, Gul S: Value of real-time endobronchial ultrasound-guided transbronchial needle aspiration. Ann Thorac Med 2011;6:77-81.

23 Navasakulpong A, Auger M, Gonzalez AV: Yield of EBUS-TBNA for the diagnosis of sarcoidosis: impact of operator and cytopathologist experience. BMJ Open Respir Res 2016; 3:e000144.

24 Casal RF, Lazarus DR, Kuhl K, NoguerasGonzález G, Perusich S, Green LK, Ost DE, Sarkiss M, Jimenez CA, Eapen GA, Morice RC, Cornwell L, Austria S, Sharafkanneh A, Rumbaut RE, Grosu H, Kheradmand F: Randomized trial of endobronchial ultrasoundguided transbronchial needle aspiration under general anesthesia versus moderate sedation. Am J Respir Crit Care Med 2015;191: 796-803.

25 van der Heijden EH, Casal RF, Trisolini R, Steinfort DP, Hwangbo B, Nakajima T, Guldhammer-Skov B, Rossi G, Ferretti M, Herth FF, Yung R, Krasnik M; World Association for Bronchology and Interventional Pulmonology, Task Force on Specimen Guidelines: Guideline for the acquisition and preparation of conventional and endobronchial ultrasound-guided transbronchial needle aspiration specimens for the diagnosis and molecular testing of patients with known or suspected lung cancer. Respiration 2014;88:500-517. 